\title{
Enhanced Nonlinear Emission from Single Multilayered Metal- Dielectric Nanocavities Resonating in the Near-Infrared
}

\author{
Nicolò Maccaferri, " Attilio Zilli, " Tommi Isoniemi, Lavinia Ghirardini, Marzia Iarossi, Marco Finazzi, \\ Michele Celebrano,* and Francesco De Angelis
}

Cite This: https://dx.doi.org/10.1021/acsphotonics.0c01500

Read Online

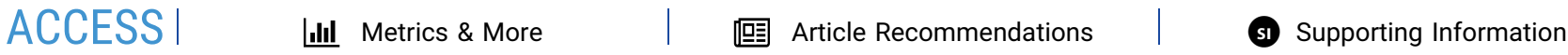

ABSTRACT: Harmonic generation mechanisms are of great interest in nanoscience and nanotechnology, since they allow generating visible light by using near-infrared radiation, which is particularly suitable for its countless applications in bionanophotonics and optoelectronics. In this context, multilayer metal-dielectric nanocavities are widely used for light confinement and waveguiding at the nanoscale. They exhibit intense and localized resonances that can be conveniently tuned in the near-infrared and are therefore ideal for enhancing nonlinear effects in this spectral range. In this work, we experimentally investigate the nonlinear emission properties of multilayer metal-dielectric nanocavities. By engineering their absorption efficiency and exploiting their intrinsic interface-induced symmetry breaking, we achieve an almost 2 orders of magnitude higher second-harmonic generation efficiency compared to gold nanostructures featuring the same geometry and optical resonant behavior. In particular, while both the third-order nonlinear susceptibility and conversion efficiency are comparable with those of the Au nanoresonators, we estimate a second-order nonlinear

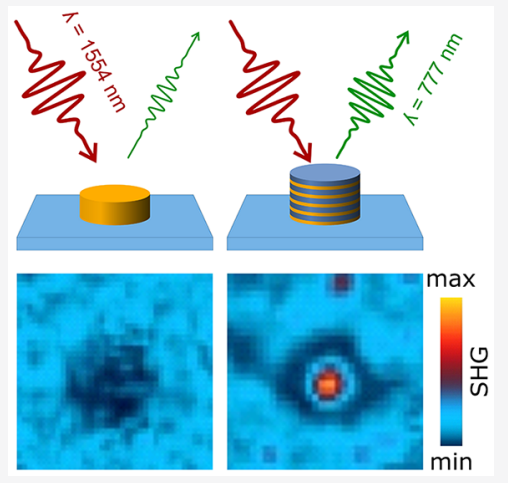
susceptibility of the order of $1 \mathrm{pm} / \mathrm{V}$, which is comparable with that of typical nonlinear crystals. We envision that our system, which combines the advantages of both plasmonic and dielectric materials, might enable the realization of composite and multifunctional nanosystems for the efficient manipulation of nonlinear optical processes at the nanoscale.

KEYWORDS: metal-dielectric nanocavities, nonlinear optics, second-harmonic generation, third-harmonic generation, multilayer, near-infrared

$M$ ultilayered metal-dielectric (MMD) cavities are widely used for light confinement and guiding at the nanoscale. So far, MMD nanocavities have successfully been implemented as negative index materials, ${ }^{1,2}$ planar waveguides, ${ }^{3,4}$ color filters, ${ }^{5}$ quantum yield enhancers, ${ }^{6-9}$ and superabsorbers driving resonant gain singularities, ${ }^{10-12}$ as well as for hotelectron generation, ${ }^{13}$ manipulation of light scattering and absorption, ${ }^{14-20}$ ultrafast all-optical switching, ${ }^{21-23}$ and highly sensitive detection. ${ }^{24,25}$ The nonlinear optical (NLO) properties of MMD systems were theoretically addressed in thin films geometries, for both second-harmonic generation $(\mathrm{SHG})^{26-29}$ and third-harmonic generation (THG). ${ }^{30}$ The main reason behind this enhanced SHG lays on the local symmetry breaking induced along the normal direction to the metaldielectric interfaces. In addition, Suresh et al. recently reported on continuous MMD thin films displaying strong Kerr nonlinearities close to their near-zero-permittivity region. ${ }^{31}$ Yet, to date, an experimental study on the NLO response of isolated MMD nanostructures is missing. In this direction, it was recently demonstrated that plasmonic nanostructures on-amirror configuration and supporting cavity gap plasmons can enable multiple and simultaneous nonlinear effects, ${ }^{32}$ a property that can be used, for instance, in multiharmonic tissue imaging to increase selectivity. ${ }^{33}$

In optics, SHG and THG mechanisms are of great interest since they generate visible light from near-infrared (NIR) radiation, which is particularly suitable for applications in biophotonics and optoelectronics. The three main reasons that make MMD nanosystems and the NIR frequency range ideal for both these applications are the following: (i) radiation with a wavelength longer than $750 \mathrm{~nm}$ falls within the transparency window of tissues and penetrates biological matter such as blood, brain/organs, and skin; ${ }^{34,35}$ (ii) dielectric materials like $\mathrm{SiO}_{2}$ display an almost flat dispersion in the NIR range, allowing to design devices that introduce minimal pulse distortion; ${ }^{36,37}$ (iii) Au nanostructures are biocompatible, easy to functionalize, and for a fundamental wavelength (FW) excitation in the NIR range, the absorption of the generated

Received: September 25, 2020 


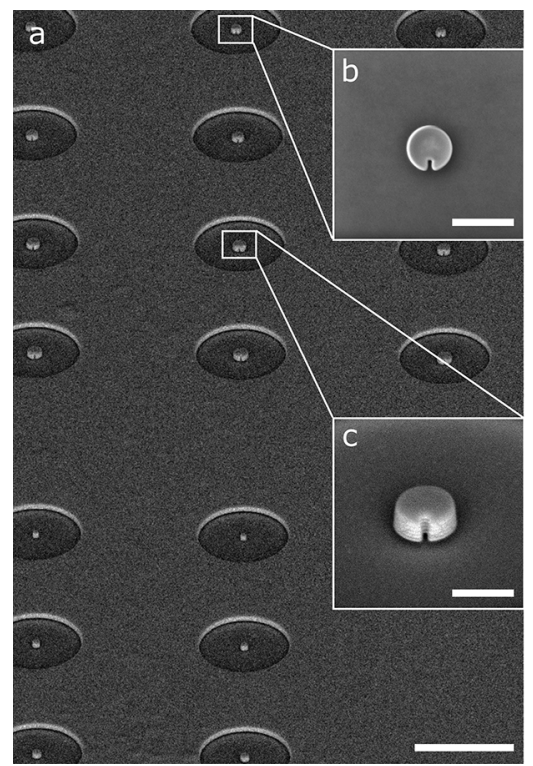

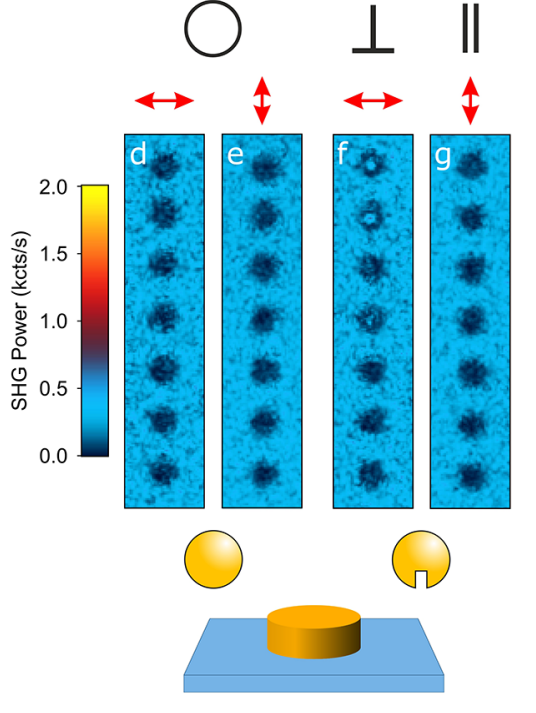

Au nanoresonator

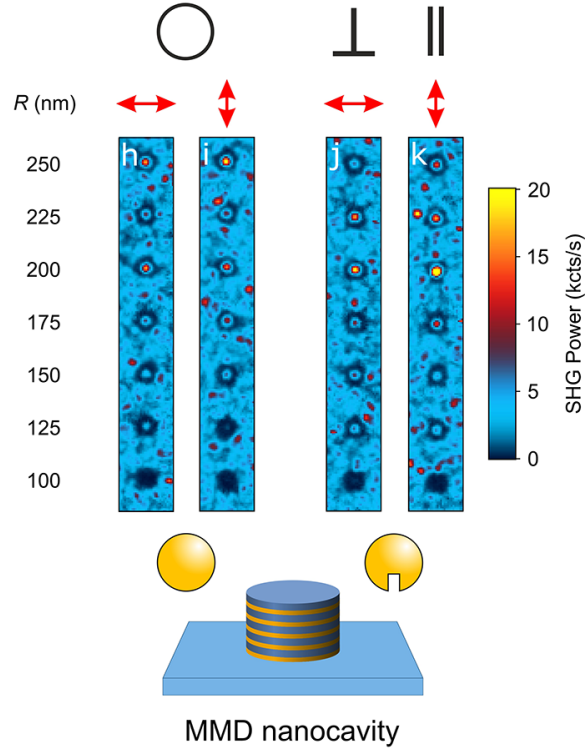

Figure 1. (a) Representative SEM images of the MMD nanocavities (scale bar $3 \mu \mathrm{m}$ ). Top (b) and side (c) views of MMD nanocavities with nominal radii of 175 and $225 \mathrm{~nm}$, respectively (scale bar in both figures is $500 \mathrm{~nm}$ ). (d-k) SHG confocal maps of Au nanoresonators (d-g) and MMD nanocavities $(\mathrm{h}-\mathrm{k})$ using a $500 \mu \mathrm{W}$ average pump power (peak intensity $1 \mathrm{GW} / \mathrm{cm}^{2}$ ). Notice that the color scales differ by 1 order of magnitude. Bright spots outside the regions where the structures are located, are related to the presence of local bumps on the sample (see also Supporting Information, Figure S3).

harmonics due to interband transitions is mitigated. Furthermore, it is well known that metal nanoparticles exhibit intense and localized field enhancements associated with plasmonic resonances, which can be conveniently tuned to the NIR, making them also ideal to enable NLO labeling in biological tissues, which in general display weaker SHG and THG signals and enhanced NLO effects in optoelectronic devices. $^{38}$

Because of the sizable values of $\chi^{(3)}$ for plasmonic metals in the visible range, the THG yield can be comparatively high in plasmonic nanoparticles at visible wavelengths. Nevertheless, the THG efficiency in $\mathrm{Au}$ is expected to significantly drop far from interband transitions, for example, when the wavelength of the pump beam is tuned to the NIR. ${ }^{39-43}$ However, it was recently demonstrated that THG can still be significantly high even when pumping in the NIR because of second-order cascade effects. ${ }^{44}$ Unfortunately, the cubic symmetry of the $\mathrm{Au}$ lattice would forbid even-order nonlinear processes, such as SHG, to take place. This limitation has been recently alleviated by engineering specific nanoantennas featuring a brokensymmetry geometry, which enables the efficient exploitation of the strong nonlinear currents developing at their surface. ${ }^{45-49}$

In this work, we address the NLO properties of metaldielectric hybrid nanosystems. The investigated platform, which can be realized also by using either chemical synthesis $^{50-52}$ or bottom-up approaches, ${ }^{53-55}$ can enhance the NLO response of the nanoantenna by engineering the absorption and scattering processes ${ }^{18}$ as well as exploiting the interface-induced local symmetry breaking typical of metaldielectric multilayers. We show that MMD nanocavities provide almost 2 orders of magnitude enhancement of the SHG response compared to pure Au nanoresonators with the same geometry and displaying plasmonic resonances in the same spectral range. We also compare their SHG and THG performance when the axial symmetry is removed with a lateral cut, realizing structures similar to those described in ref 49 , and show that, in our experiments, such symmetry breaking plays a minor role compared to the pivotal effect of the multilayered structure in enhancing NLO effects.

\section{RESULTS AND DISCUSSION}

MMD nanocavities made of five layers of $\mathrm{Au}(15 \mathrm{~nm})$ alternating with five layers of $\mathrm{SiO}_{2}(15 \mathrm{~nm})$ were fabricated using gallium focused ion beam (FIB) milling (see Methods for more details) and designed to exhibit strong absorption in the NIR spectral range, as previously demonstrated in ref. [18]. The nominal radius $R$ of the structures spans from 100 to $250 \mathrm{~nm}$ in steps of $25 \mathrm{~nm}$. Each structure was realized in several nominally identical replicas to check the reproducibility of the NLO properties of the single nanostructures (see Figure 1a). For comparison, we fabricated also bulk $\mathrm{Au}$ nanoresonators with the same amount of gold as in the MMD nanocavities (thickness $75 \mathrm{~nm}$ ) and resonating in the same spectral range (see Supporting Information Figure S1). For both MMD and bulk Au systems we fabricated an additional set of nanostructures with a radial cut, which removes the axial symmetry. The insertion of a lateral cut lifts the axial symmetry and induces a doubly resonant character in the scattering spectrum at both the FW and second harmonic ( $\mathrm{SH}$ ) wavelength. Therefore, it is expected to improve the outcoupling of the $\mathrm{SH}$ sources inside the nanostructure to the radiating modes. ${ }^{49}$ Figure $1 \mathrm{a}-\mathrm{c}$ shows representative scanning electron microscopy (SEM) images of the MMD nanocavities with the radial cut. The nanostructures were obtained by milling a continuous multilayered film (or bulk gold) evaporated on a glass substrate and are encircled by a trench dug into the same film, which has been used as reference.

To assess separately the effect of multilayering and of symmetry removal, we collected SHG maps on both bulk Au nanoresonators and MMD nanocavities with axial symmetry (i.e., disc shape, from now on indicated with the symbol " $O$ ") and on structures with a cut. The latter were measured for different exciting polarizations, namely, with the FW electric 

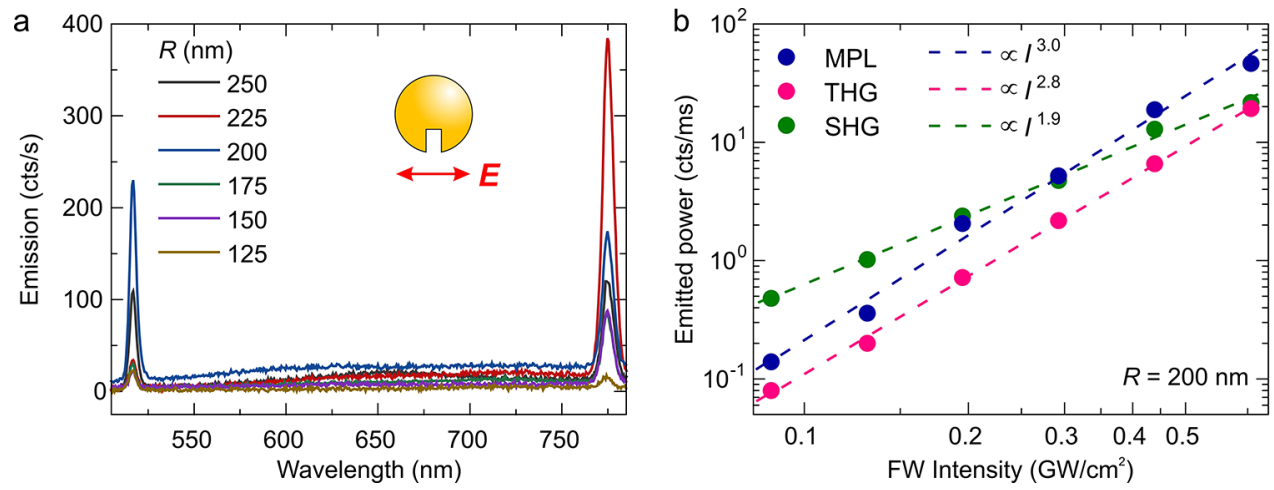

Figure 2. (a) NLO emission spectra by single cut structures of different radii as indicated in the legend. Excitation at the FW (1554 nm), “ $\perp$ ” configuration. (b) NLO emission as a function of power by a single cut structure of radius $R=200 \mathrm{~nm}$. The signal is optically filtered using narrowband bandpass filters (center/bandwidth in $\mathrm{nm} \mathrm{775/25}$ for SHG, 520/40 for THG) and a combination of long-pass (cut-on at 550 nm) and short-pass (cut-off at $750 \mathrm{~nm}$ ) filters for the MPL. The dashed lines are power fits to the data, with exponents indicated in the legend.

field either parallel (“\|” configuration) or perpendicular (“ $\perp ”$ configuration) to the cut.

NLO measurements were performed on single nanostructures (see Figure $1 \mathrm{~d}-\mathrm{k}$ ) by tightly focusing a $500 \mu \mathrm{W}$ average pump power, which is equivalent to a peak intensity of about $1 \mathrm{GW} / \mathrm{cm}^{2}$, using a confocal microscope coupled to a pulsed laser at a telecom wavelength $(\lambda=1554 \mathrm{~nm})$. The setup, which is similar to that described in ref 44 , is illustrated in more detail in the Methods section and in Supporting Information, Figure S2. While the SHG collected from Au nanoresonators (Figure $1 \mathrm{~d}-\mathrm{g})$ is extremely weak, MMD nanocavities, both with (Figure $1 \mathrm{j}, \mathrm{k}$ ) and without the radial cut (Figure $1 \mathrm{~h}, \mathrm{i}$ ), produce a steady SHG. In particular, in our experimental conditions, the "O" bulk Au structures do not yield any detectable SHG signal (Figure $1 \mathrm{~d}, \mathrm{e})$. This is one more reason why we have also realized structures with a radial cut, which brings about a sizable SHG even in the Au nanoresonators under " $\perp$ " illumination (Figure 1f). On the other hand, the SHG signal of bulk Au cut discs is not detected with our instrumentation under "I" illumination (Figure 1g).

For the MMD structures, the behavior is different: the SHG yield increases significantly also for the structures without the lateral cut (i.e., with axial symmetry) and a resonant behavior appears in the maps (see Figure $1 \mathrm{~h}-\mathrm{k}$ ). We have estimated the SHG signal enhancement by evaluating the count rates in the maps collected on either the bulk $\mathrm{Au}$ or the MMD nanostructures with the radial cut under " $\perp$ " illumination. At $500 \mu \mathrm{W}$ average pump power (peak intensity $1 \mathrm{GW} / \mathrm{cm}^{2}$ ), we measured a count rate of $0.6 \mathrm{kcts} / \mathrm{s}$ from the most efficient $\mathrm{Au}$ nanoresonator (the second from the top in Figure if) compared to $21 \mathrm{kcts} / \mathrm{s}$ from the most efficient MMD nanocavity (the third from the top in Figure 1j). This corresponds to an enhancement factor of about 35 for the MMD structures with respect to bulk Au structures. Under "I" illumination the count rate for the most efficient MMD is $42 \mathrm{kcts} / \mathrm{s}$. Although the assessment of the enhancement in this configuration is prevented by the unknown SHG signal on the bulk Au structures, by assuming the signal of the homogeneous $\mathrm{Au}$ nanoresonators to be of the order of the noise level, we can estimate a lower bound value for the enhancement factor that is as high as 70. The same can be applied to the "O" geometry, which yields $15 \mathrm{kcts} / \mathrm{s}$ in the MMD case, but no signal for the $\mathrm{Au}$ nanoresonators. Concurrently, the enhancement factor induced by the layering alone, which is obtained by comparing the SHG yield from the bulk $\mathrm{Au}$ and MMD film
( 0.5 and $6 \mathrm{kcts} / \mathrm{s}$, respectively), is about 10 . We thus ascribe the enhancement to the combination of two main mechanisms: (i) the appearance of a narrow absorption peak at the fundamental wavelength typical of these MMD nanocavities ${ }^{18}$ (see also Supporting Information, Figure S1) and (ii) the local inversion symmetry-breaking at the metal-dielectric interfaces. Therefore, we conclude that shaping the MMD into resonant nanoparticles allows one to further boost the enhancement by over a factor of 3 while considerably reducing the material fingerprint. Although the presence of the radial cut in the MMD structure introduces a further symmetry breaking in the axial symmetry, it does not provide a significant enhancement of the SHG with respect to multilayered "O" shaped nanostructures. This is attributed to the $\mathrm{Au}-\mathrm{SiO}_{2}$ layering, which dominates over the effects induced by the axial symmetry breaking and the field enhancement in the cut.

By resolving the NLO emission spectrum of the individual nanocavities of different radii in the visible range (see Figure 2a), it is possible to observe, in addition to the strong SHG peak at $777 \mathrm{~nm}$, a THG peak at $518 \mathrm{~nm}$, as well as a sizable and spectrally broad multiphoton photoluminescence (MPL) band typical of $\mathrm{Au}^{56,57}$ The emission spectra in the "O" and "Il" cases exhibit similar features (see Supporting Information, Figure S4). To complete the characterization of the NLO behavior, we recorded the power dependence of SHG, THG, and MPL from a resonant nanostructure $(R=200 \mathrm{~nm}$, “ $\perp$ " case, Figure $2 b$ ) reported in Figure 2a, singling out each NLO emission signal by spectral filtering. The incident intensity on the sample, I, was varied by almost an order of magnitude, and the emitted power $P$ fitted by a power function $P=\operatorname{cost} \cdot I^{\alpha}$. While the power coefficients $\alpha=1.9$ and 2.8 closely match the quadratic and cubic dependence expected for SHG and THG, respectively, the power coefficient determined for MPL is $\alpha=$ 3.0, indicating a three-photon absorption process. ${ }^{58,59}$ This small discrepancy is within the experimental uncertainties associated with characterizations of single particle NLO properties, which can be attributed to residual background that might affect more the measurements at lower powers, hence, decreasing the slope.

Considering the SHG and THG emission by the most efficient MMD nanocavity in the experiment (i.e., $R=200 \mathrm{~nm}$ ) and accounting for the optical losses in the detection path to retrieve the emitted power, we experimentally estimate the following effective values of the second- and third-order nonlinear susceptibilities, $\chi^{(2)}$ eff and $\chi^{(3)}$ eff (see Supporting 

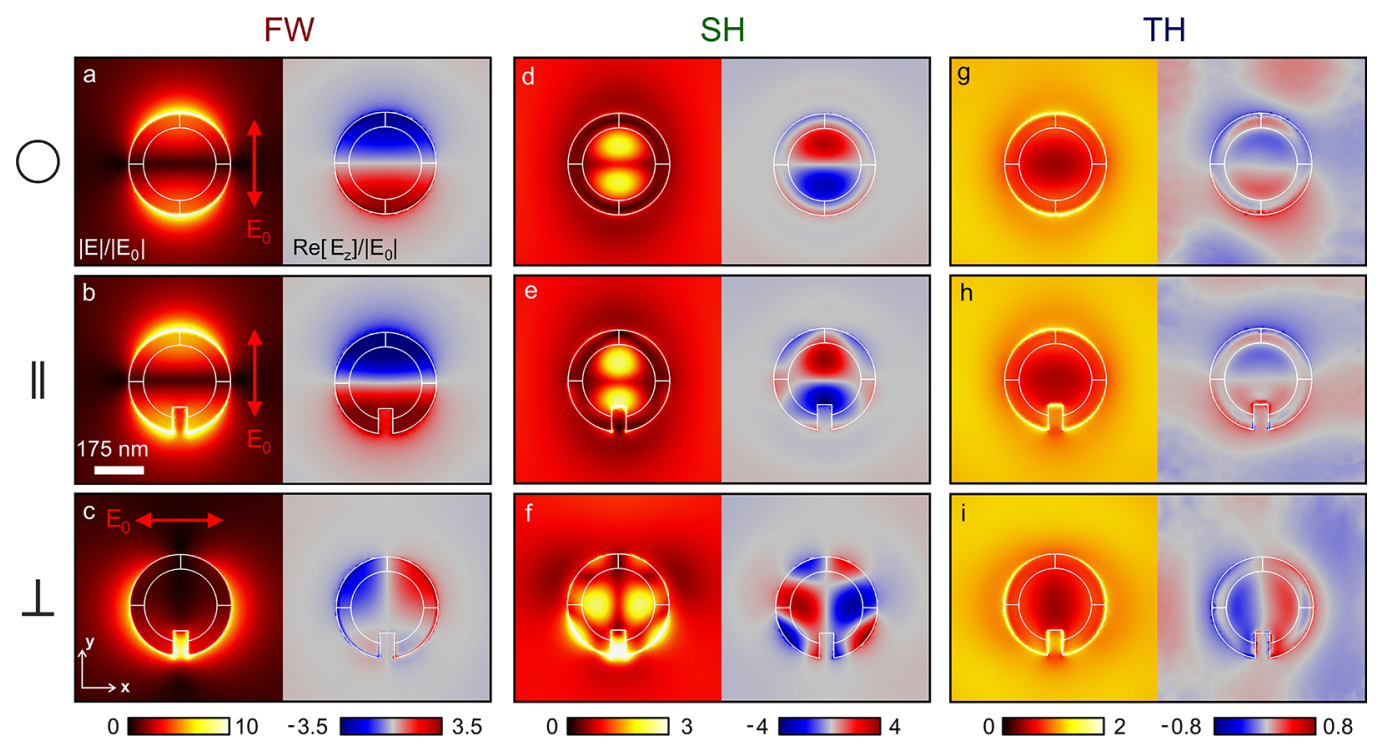

Figure 3. Simulated in-plane near field profiles of a MMD nanocavity with $R=175 \mathrm{~nm}$. Panels (a), (b), and (c) are the computed near fields at the FW (1554 nm) for the $O, \|$, and $\perp$ cases. Panels (d), (e), and (f) are the computed near-fields at the SH (777 nm) for the $O, \|$, and $\perp$ cases. Panels $(\mathrm{g}),(\mathrm{h})$, and (f) are the computed near fields at the TH $(518 \mathrm{~nm})$ for the $\mathrm{O}, \|$, and $\perp$ cases. In each panel, the figures on the left show the electric field amplitude normalized to the incident plane wave amplitude $\left|E_{0}\right|$, while the figures on the right show the real part of the $z$-component of the electric field amplitude normalized to the incident plane wave amplitude $\left|E_{0}\right|$.
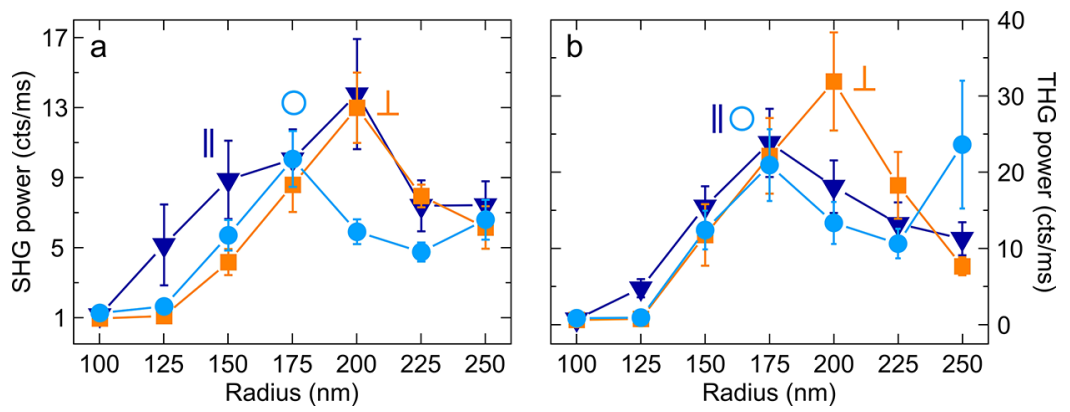

Figure 4. SHG (a) and THG (b) power (most intense pixel of the confocal image of the structure) of the MMD nanocavities. Each plotted point is the mean measured over 10 nominally identical replicas, and the error bars correspond to the standard error of the mean values. The signals are collected using an illumination average power of about $500 \mu \mathrm{W}$ (peak intensity $1 \mathrm{GW} / \mathrm{cm}^{2}$ ).

Information, Note 1$): \chi^{(3)}{ }_{\text {eff }} \approx 10^{-20} \mathrm{~m}^{2} / \mathrm{V}^{2}$, in line with THG measurements on $\mathrm{Au}$ in this wavelength range, ${ }^{42}$ and $\chi_{\text {eff }}^{(2)} \approx 1.5 \mathrm{pm} / \mathrm{V}$, which is comparable to some widely employed second-order upconverting nonlinear crystals, such as potassium dideuterium phosphate (KDP). ${ }^{60}$ Moreover, we found for the best MMD nanocavity a conversion efficiency $\eta_{\mathrm{SHG}}=\frac{P_{\mathrm{SHG}}^{\text {out }}}{P_{\mathrm{FW}}^{\text {in }}} \cong 6 \times 10^{-10}$, whereas for the best gold nanoresonator measured in the same experiment $\eta_{\mathrm{SHG}}$ is of the order $10^{-11}$. For THG, we found $\eta_{\mathrm{THG}}=\frac{P_{\mathrm{THG}}^{\text {out }}}{P_{\mathrm{FW}}^{\text {in }}} \cong 1.8 \times 10^{-10}$, which is comparable to that of gold.

To gain further insight into the optical properties of the MMD nanocavities studied in this work, we simulated numerically their near-field response using the finite element method (see Methods for additional details). Figure 3 shows the simulated enhancement of the electric field amplitude $\left|E / E_{0}\right|$ at the $\mathrm{FW}, \mathrm{SH}$, and third-harmonic $(\mathrm{TH})$ wavelength by a single nanocavity with $R=175 \mathrm{~nm}$, which corresponds to the most efficient "O" structure. Following the nanostructure design, the largest enhancement is observed at the FW, whose field contributes quadratically to the SHG process and cubically to the THG one. ${ }^{61}$ This enhancement is associated with the onset of an absorption peak, typical of these MMD systems at telecom wavelengths (see also Supporting Information, Figure S1). The field is mostly localized in the dielectric layers at the $\mathrm{SH}$ wavelength, and $\mathrm{SHG}$ is expected to occur mostly at the $\mathrm{Au}-\mathrm{SiO}_{2}$ interface, where the inversion symmetry of the two materials is broken. Conversely, at the $\mathrm{TH}$ wavelength the field is mostly confined outside the structure and at the metal surface, which has the highest $\chi^{(3)}$ and generates most of the $\mathrm{TH}$ emission.

The spatial distribution of the mode excited at the FW is governed by a bonding dipolar resonance, as previously demonstrated in ref 18 . Notably, the presence of a cut inducing the in-plane symmetry breaking makes little difference with respect to the " $O$ " case (Figure 3a) under "II" illumination (Figure 3b). Apart from a slight variation of the field distribution, this also holds true at the $\mathrm{SH}$ and $\mathrm{TH}$ wavelengths for both " $O$ " (Figure 3d,g) and II (Figure 3e,h) cases. On the contrary, the symmetry breaking significantly modifies the spatial field distribution under " $\perp$ " illumination at the FW (Figure 3c), SH (Figure 3f), and TH (Figure 3i) wavelength. In particular, strong light confinement occurs at the cut edges at the $\mathrm{SH}$ wavelength (Figure $3 \mathrm{f}$ ). This analysis 


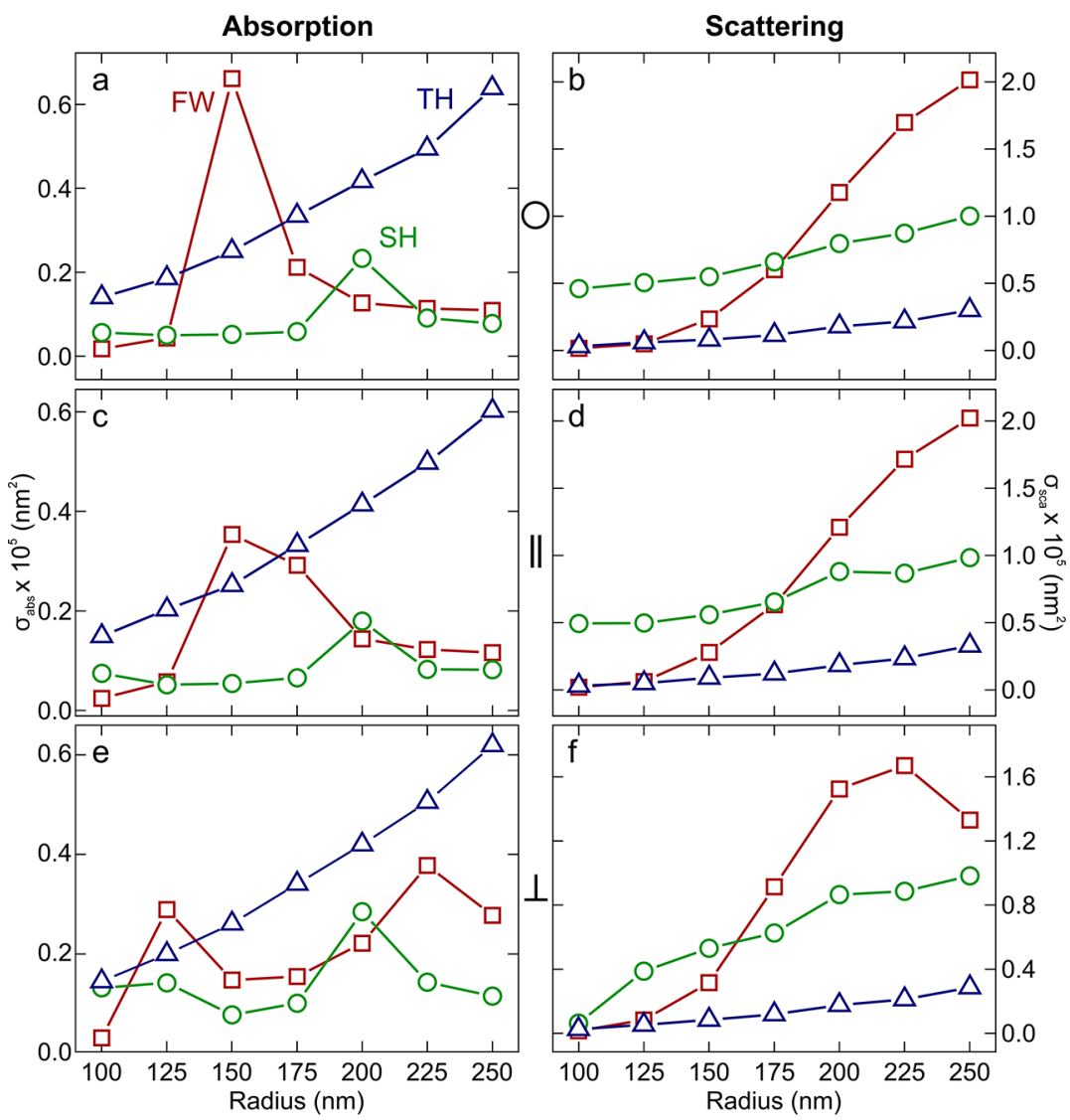

Nonlinear efficiencies $\left(\epsilon^{(x)}\right)$
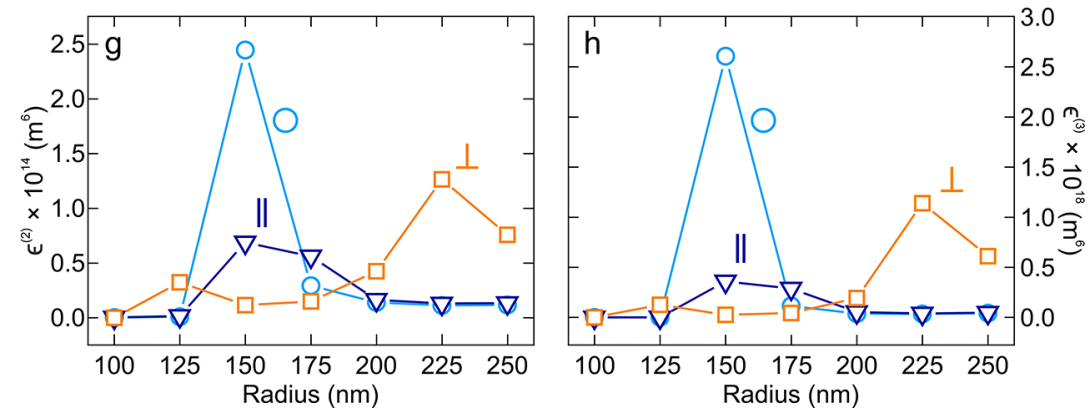

Figure 5. Calculated absorption (left) and scattering (right) cross sections for the "O” (a, b), “\|” (c, d) and " $\perp$ ” (e, f) cases, as a function of the structure radius at the FW (brown curves), SH (green curves) and TH (blue curves). Calculated (g) efficiencies $\epsilon^{(2)}$ and (h) $\epsilon^{(3)}$ as a function of the nanocavity radius.

predicts a similar NLO response in the "O" and " $\mid$ " cases, while some differences are expected for the “ $\perp$ ” case.

To evaluate the effect of the geometry, we performed a systematic study of the NLO response of the individual MMD nanocavities with radius increasing from 100 to $250 \mathrm{~nm}$ in steps of $25 \mathrm{~nm}$. The measured SHG and THG powers, averaged over 10 nominally identical replicas of each structure, are reported in Figure $4 a$ and b, respectively, for all structures and excitation configurations. In Figure 4a,b, the sizable error bars, representing the standard error of the mean, likely reflect the role of random defects introduced by the nanofabrication process, such as roughness in the thin films due to electronbeam deposition and radiation damage induced by the gallium beam.

In the " $O$ " structure, the NLO emission response is similar for both SHG and THG with a maximum obtained for a radius of $175 \mathrm{~nm}$, indicating that the emission is dominated by the resonant behavior at the FW. The cut nanostructures exhibit a more complex behavior. The SHG signal under both $\|$ and $\perp$ illumination features a maximum shifted toward longer radii $(200 \mathrm{~nm})$. Conversely, the THG peak for the "\|" case is found for the same radius $(175 \mathrm{~nm})$ as the "O" case, in agreement with the similar spatial distribution of the calculated near-field distributions in Figure 3. Conversely, the THG emission under $\perp$ illumination peaks for the same radius $(200 \mathrm{~nm})$ that maximizes its SHG signal. The perfect match between the SHG peaks of the "\|l" and " $\perp$ " cases at $R=200 \mathrm{~nm}$ is a clear indication that SHG does not depend on the localized fields in the cut or on the consequent axial symmetry breaking. On the other hand, the shoulder in the SHG response for shorter radii in the $\|$ configuration is unexpected and could be related to local random defects of the nanostructured MMDs.

To better assess the mechanisms underpinning SHG and THG, we numerically computed the scattering and absorption 
cross sections $\sigma_{\text {sca }}$ and $\sigma_{\text {abss }}$, at the FW, SH, and $\mathrm{TH}$ wavelengths for all the nanostructure cases (Figure 5a-f). By inspecting the radius dependence of $\sigma_{\mathrm{sca}}$ and $\sigma_{\mathrm{abs}}$ at the FW, the "O" and "I" cases (red curves in Figure 5a,b and Figure 5c,d, respectively) share a similar trend, whereas in the " $\perp$ ” case (red curves in Figure 5e,f) we observe a different behavior, as expected also from the near-field simulations. In particular, both $\sigma_{\text {abs }}$ and $\sigma_{\text {sca }}$ peak at $R=225 \mathrm{~nm}$, and this could explain the shift of both the SHG and THG peak efficiencies toward larger $R$ values with respect to the " $O$ " case.

A simple approach to model the nonlinear emission from these structures, ultimately based on the Miller's rule, ${ }^{62,63}$ is to combine the calculated radius-dependent linear cross sections (Figure 5a-f) into the NLO coefficients $\epsilon^{(2)} \equiv$ $\sigma_{\text {abs }}(\omega)^{2} \sigma_{\text {sca }}(2 \omega)$ and $\epsilon^{(3)} \equiv \sigma_{\text {abs }}(\omega)^{3} \sigma_{\text {sca }}(3 \omega)$, assuming that the NLO process is governed by absorption at the FW wavelength and by scattering at the harmonic emission wavelength (Figure $5 \mathrm{~g}, \mathrm{~h}$ ). These coefficients account for the coupling strength between the antenna and the radiation at the excitation and emission wavelengths. This description of the process does not consider the spatial overlap between the fields ${ }^{47}$ at the two wavelengths of interest (which can be qualitatively assessed from Figure 3) and the relative polarization enabled by the selection rules for the NLO processes. The measured NLO emission trends in Figure $4 \mathrm{a}, \mathrm{b}$ are in qualitative agreement with the computed nonlinear coefficients (see Figure 5g,h). In particular, these coefficients allow to reproduce the experimental trend of THG in all the configurations, also predicting the shift of THG toward larger radii introduced by the cut for " $\perp$ " illumination. Moreover, by comparing Figure $4 \mathrm{~b}$ with Figure $5 \mathrm{~h}$, it is evident that, despite small shifts and some broadening in the experimental results, the trend of $\epsilon^{(3)}$ mirrors the absorption resonances at the FW (see also Figure 5a,c,e). This demonstrate that the intuitive model adopted is effective in reproducing the nonlinear processes based on dipole-allowed transitions such as THG, that is, for a diagonal nonlinear susceptibility tensor. Contrariwise, while the $\mathrm{SH}$ emission is well reproduced for both the " $O$ " and " $\perp$ " case, revealing the major role played by the resonance at the FW like in theTHG, the model (see Figure $5 \mathrm{~g}$ ) fails to fully grasp the behavior of the SHG for the "\|" case (see Figure 4a). To this aim, we have evaluated surface overlap integrals between the FW and $\mathrm{SH}$ fields for all the configurations, after taking into account (i) the selection rules for the SHG process according to symmetry, ${ }^{64}$ and (ii) the surface character of the process.

However, this model refinement does not yield a better agreement with the experimental data than the one provided by the NLO coefficient estimated above. We ascribed this inconsistency to the fact that in SHG the colocalization and copolarization of the electric fields at the FW and $\mathrm{SH}$ becomes more relevant within the structure at the interfaces between the multilayers, rather than within the cut (see Figure 3b,e). This mechanism, which cannot be easily included in our model and, possibly, even into a more refined nonlinear model, can be underpinned by surface roughness at the interfaces between layers, while the removal of the axial symmetry reduces the destructive interference of the $\mathrm{SH}$ radiation in the far field. ${ }^{47}$

\section{CONCLUSION}

We reported efficient second- and third-harmonic emission from multilayered metal-dielectric nanocavities, where the absorption at the fundamental wavelength is enhanced by tailoring the geometry of the structures. We proved that the presence of multiple metal-dielectric interfaces, rather than the breaking of the axial-symmetry of the nanocavities, boosts the NLO generation. As a result, the NLO response is largely independent from the exciting polarization, confirming the results reported in ref 19 . Noticeably, we found that in our experimental conditions the second-harmonic emission is almost 2 orders of magnitude larger than that of pure $\mathrm{Au}$ nanostructures with the same geometry and optical resonant behavior. We underline that these structures can be fabricated on a large scale by using hole-mask colloidal lithography also to achieve complicated structures ${ }^{18,53-55,65}$ and detached from the substrate. Although, out of the scope of this work, we foresee that these structures can be used as nonlinear probes in biological systems, where it is fundamental to control the emission of visible light from objects that resonate in the NIR spectral range and are located deep in the tissue. In this context, the choice of materials is important to provide high values of $\chi^{(2)}$ and $\chi^{(3)}$. We have found that our nonlinear susceptibilities are of the same order of magnitude as common nonlinear optical crystals, such as KDP. Other materials, such as lithium niobate $\left(\mathrm{LiNbO}_{3}\right)$, potassium dihydrogen phosphate $\left(\mathrm{KH}_{2} \mathrm{PO}_{4}\right)$, barium titanate $\left(\mathrm{BaTiO}_{3}\right)$, and bismuth ferrite $\left(\mathrm{BiFeO}_{3}\right)$, though having higher $\chi^{(2)}$ values, are not well-suited for in vivo imaging due to their cytotoxicity. ${ }^{66,67}$ On the contrary, Au nanoparticles and dielectric materials such as $\mathrm{SiO}_{2}$ are widely used in nanomedicine thanks to their biocompatibility. ${ }^{68}$ Thus, our approach is a first step toward merging the advantages of biocompatible plasmonic materials with those offered by the emerging field of soft polymers ${ }^{69}$ by realizing composite and multifunctional nanosystems. Finally, a very exciting perspective for these multilayered architectures would be to combine their nonlinear optical properties with those of layered materials. $^{22,70}$ A step toward this direction has been already taken by some groups, for instance, by coupling multilayered metal-dielectric nanocavities with quantum emitters $^{7}$ or $\mathrm{WS}_{2}$ monolayers. ${ }^{71}$

\section{METHODS}

Sample Fabrication. The multilayers were deposited on a glass substrate via electron-beam evaporation (Kurt J. Lesker PVD75). The $1 \mathrm{~mm}$ thick glass slide was cleaned with acetone, isopropanol, and oxygen plasma. The layers were deposited sequentially, starting with $15 \mathrm{~nm}$ of $\mathrm{Au}$ and continuing with $15 \mathrm{~nm}$ of $\mathrm{SiO}_{2}$ for five layers of each, and capped with an additional protective layer of $50 \mathrm{~nm} \mathrm{SiO}{ }_{2}$. The pillar structures were created with focused ion beam milling using a gallium beam (FEI Helios NanoLab 650 dual beam system) at $24 \mathrm{pA}$, milling down to the glass surface around each pillar. The empty milled area around each structure was a circle $3 \mu \mathrm{m}$ in diameter, and the required milling time was $6 \mathrm{~min}$ per structure. The pillars have a radius varying from 100 to $250 \mathrm{~nm}$ in steps of $25 \mathrm{~nm}$, and the variant with cuts has nominally $50 \mathrm{~nm}$ wide, half-radius long radial cuts. The control samples were fabricated by depositing $75 \mathrm{~nm}$ of $\mathrm{Au}$, namely, the same amount as for the multilayers, followed by a top layer of $50 \mathrm{~nm}$ of $\mathrm{SiO}_{2}$, and then, realizing disks with FIB in a similar way as described for the MMD disks. The structures were imaged with scanning electron microscopy in the same system after milling.

Numerical Simulations. Calculations using the finite element method have been performed using Comsol Multiphysics, a commercial solver. The refractive index values of 
gold and $\mathrm{SiO}_{2}$ have been taken from literature. ${ }^{36,72}$ To simulate the optical properties of MMD nanocavities we have considered a simulation region $(2 \mu \mathrm{m} \times 2 \mu \mathrm{m} \times 4 \mu \mathrm{m})$, where we specified the exciting ("background") electric field (a linearly polarized plane wave, intensity of the incident light $I_{0}=$ $1 \mathrm{GW} / \mathrm{cm}^{2}$ ), and then we calculated the scattered field by a single nanocavity to extract observables such as the optical absorption and scattering cross sections, by using the procedure reported in this example from Comsol. ${ }^{73}$ The scattering cross-section is defined as $\sigma_{\text {sca }}=\frac{1}{I_{0}} \iint(\mathbf{n} \cdot \mathbf{S}) \mathrm{d} S$, where $\mathbf{n}$ is the unit vector normal to the integration surface pointing outward and $\mathbf{S}$ is the Poynting vector. The integral is taken over the closed surface of the meta-antenna. The absorption cross section equals $\sigma_{\mathrm{abs}}=\frac{1}{I_{0}} \iiint Q \mathrm{~d} V$, where $Q$ is the resistive power loss density of the system and the integral is taken over the volume of the nanocavity.

Nonlinear Optical Experiments. The nonlinear optical measurements were performed on single nanostructures using a confocal microscope coupled with a laser delivering ultrafast pulses $\left(\Delta \tau_{\text {pulse }}=160 \mathrm{fs}, f_{\text {rep }}=80 \mathrm{MHz}\right)$ centered at $1554 \mathrm{~nm}$. The pump beam is focused using a 0.85 NA air objective, which produces a $2.2 \mu \mathrm{m}$ beam diameter. All the experiments except the power-dependence study (Figure 2b) were performed with $500 \mu \mathrm{W}$ average pump power at the sample (peak intensity $1 \mathrm{GW} / \mathrm{cm}^{2}$ ). The nonlinear emission is then collected and sent either to a single-photon avalanche diode (SPAD) detector or to a spectrometer (Andor, Shamrock SR303i) equipped with a CCD camera (Andor, iKon-M DU934P-BV) to acquire the emission spectrum. The power curves of SHG, THG, and MPL reported in Figure $2 \mathrm{~b}$ were obtained by singling out each nonlinear emission signal by spectral filtering and detecting through the SPAD detector.

\section{ASSOCIATED CONTENT}

\section{SI Supporting Information}

The Supporting Information is available free of charge at https://pubs.acs.org/doi/10.1021/acsphotonics.0c01500.

Calculated scattering and absorption cross sections of MMD nanocavities and bulk Au nanoresonators. Sketch of the nonlinear microscope used in the experiments. SEM and dark field images of a typical region of the MMD sample. Emission spectra recorded for " $O$ " and "|l” MMD nanocavities for different radii. Real part of the $z$-component of the electric field at the fundamental, $\mathrm{SH}$ and $\mathrm{TH}$ wavelength for different MMD nanocavity radii. Note on the estimation of the SHG and THG conversion efficiencies, nonlinear coefficients, and nonlinear susceptibilities (PDF)

\section{AUTHOR INFORMATION}

\section{Corresponding Author}

Michele Celebrano - Department of Physics, Politecnico di Milano, I-20133 Milano, Italy; (1) orcid.org/0000-00033336-3580; Email: michele.celebrano@polimi.it

\section{Authors}

Nicolò Maccaferri - Department of Physics and Materials Science, University of Luxembourg, L-1511 Luxembourg, Luxembourg; ○ orcid.org/0000-0002-0143-1510

Attilio Zilli - Department of Physics, Politecnico di Milano, I20133 Milano, Italy; ㅇo orcid.org/0000-0003-1845-6850
Tommi Isoniemi - Department of Physics and Astronomy, University of Sheffield, Sheffield S3 7RH, United Kingdom; Istituto Italiano di Tecnologia, I-16163 Genova, Italy; (1) orcid.org/0000-0003-0238-1006

Lavinia Ghirardini - Department of Physics, Politecnico di Milano, I-20133 Milano, Italy

Marzia Iarossi - Istituto Italiano di Tecnologia, I-16163 Genova, Italy; Dipartimento di Informatica, Bioingegneria, Robotica e Ingegneria dei Sistemi (DIBRIS), Università degli Studi di Genova, I-16126 Genova, Italy

Marco Finazzi - Department of Physics, Politecnico di Milano, I-20133 Milano, Italy; @orcid.org/0000-0002-9197-3654

Francesco De Angelis - Istituto Italiano di Tecnologia, I16163 Genova, Italy; 이이.org/0000-0001-6053-2488

Complete contact information is available at:

https://pubs.acs.org/10.1021/acsphotonics.0c01500

\section{Author Contributions}

${ }^{\#}$ N.M. and A.Z. contributed equally to this work. N.M. and M.C. conceived the study. N.M. designed the structures and performed the calculations with inputs from A.Z., M.F., and M.C. A.Z., L.G., and M.C. designed the experiments and performed the nonlinear optical response characterization. N.M., A.Z., M.F., and M.C. analyzed the data, interpreted the results, and wrote the manuscript. T.I., M.I., and F.D.A. fabricated and imaged the samples and participated in the general discussion.

\section{Notes}

The authors declare no competing financial interest.

\section{ACKNOWLEDGMENTS}

N.M. acknowledges support from the Luxembourg National Research Fund (Grant No. C19/MS/13624497 "ULTRON”) and from the FEDER Program (Grant No. 2017-03-022-19 "Lux-Ultra-Fast"). A.Z., M.F., and M.C. acknowledge financial support by the Italian Ministry of University and Research (MIUR) through the PRIN Project "NOMEN" (Prot. 2017MP7F8F).

\section{REFERENCES}

(1) Lezec, H. J.; Dionne, J. A.; Atwater, H. A. Negative refraction at visible frequencies. Science 2007, 316, 430-432.

(2) Caligiuri, V.; Palei, M.; Imran, M.; Manna, L.; Krahne, R. Planar double-epsilon-near-zero cavities for spontaneous emission and Purcell effect enhancement. ACS Photonics 2018, 5, 2287-2294.

(3) Dionne, J. A.; Sweatlock, L. A.; Atwater, H. A.; Polman, A. Planar metal plasmon waveguides: frequency-dependent dispersion, propagation, localization, and loss beyond the free electron model. Phys. Rev. B: Condens. Matter Mater. Phys. 2005, 72, 075405.

(4) Maccaferri, N.; Isoniemi, T.; Hinczewski, M.; Iarossi, M.; Strangi, G.; De Angelis, F. Designer Bloch plasmon polariton dispersion in grating-coupled hyperbolic metamaterials. APL Photonics 2020, 5, 076109.

(5) Li, Z.; Butun, S.; Aydin, K. Large-area, lithography-free super absorbers and color filters at visible frequencies using ultrathin metallic films. ACS Photonics 2015, 2, 183-188.

(6) Guclu, C.; Luk, T. S.; Wang, G. T.; Capolino, F. Radiative emission enhancement using nano-antennas made of hyperbolic metamaterial resonators. Appl. Phys. Lett. 2014, 105, 123101.

(7) Indukuri, S. R. K. C.; Bar-David, J.; Mazurski, N.; Levy, U. Ultrasmall Mode Volume Hyperbolic Nanocavities for Enhanced Light-Matter Interaction at the Nanoscale. ACS Nano 2019, 13, 11770-11780. 
(8) Lu, D.; Qian, H.; Wang, K.; Shen, H.; Wei, F.; Jiang, Y.; Fullerton, E. E.; Yu, P. K. L.; Liu, Z. Nanostructuring Multilayer Hyperbolic Metamaterials for Ultrafast and Bright Green InGaN Quantum Wells. Adv. Mater. 2018, 30, 1706411.

(9) Lu, D.; Kan, J. J.; Fullerton, E. E.; Liu, Z. Enhancing spontaneous emission rates of molecules using nanopatterned multilayer hyperbolic metamaterials. Nat. Nanotechnol. 2014, 9, 48-53.

(10) Zhou, J.; Kaplan, A. F.; Chen, L.; Guo, L. J. Experiment and Theory of the Broadband Absorption by a Tapered Hyperbolic Metamaterial Array. ACS Photonics 2014, 1, 618-624.

(11) Caligiuri, V.; Pezzi, L.; Veltri, A.; De Luca, A. Resonant Gain Singularities in 1D and 3D Metal/Dielectric Multilayered Nanostructures. ACS Nano 2017, 11, 1012-1025.

(12) Riley, C. T.; Smalley, J. S. T.; Brodie, J. R. J.; Fainman, Y.; Sirbuly, D. J.; Liu, Z. Near-perfect broadband absorption from hyperbolic metamaterial nanoparticles. Proc. Natl. Acad. Sci. U. S. A. 2017, 114, 1264-1268.

(13) Sakhdari, M.; Hajizadegan, M.; Farhat, M.; Chen, P.-Y. Efficient, broadband and wide-angle hot-electron transduction using metal-semiconductor hyperbolic metamaterials. Nano Energy 2016, 26, 371-381.

(14) Wang, P.; Krasavin, A. V.; Viscomi, F. N.; Adawi, A. M.; Bouillard, J.-S. G.; Zhang, L.; Roth, D. J.; Tong, L.; Zayats, A. V. Metaparticles: Dressing Nano-Objects with a Hyperbolic Coating. Laser \& Photonics Reviews 2018, 12, 1800179.

(15) Tulegenov, D.; Valagiannopoulos, C. Uniaxial films of maximally controllable response under visible light. Sci. Rep. 2020, 10,13051

(16) Zhumabek, T.; Valagiannopoulos, C. Light trapping by arbitrarily thin cavities. Physical Review Research 2020, 2, 043349.

(17) Song, J.; Zhou, W. Multiresonant Composite Optical Nanoantennas by Out-of-plane Plasmonic Engineering. Nano Lett. 2018, 18, 4409-4416.

(18) Isoniemi, T.; Maccaferri, N.; Ramasse, Q. M.; Strangi, G.; De Angelis, F. Electron Energy Loss Spectroscopy of Bright and Dark Modes in Hyperbolic Metamaterial Nanostructures. Adv. Opt. Mater. 2020, 8, 2000277.

(19) Maccaferri, N.; Zhao, Y.; Isoniemi, T.; Iarossi, M.; Parracino, A.; Strangi, G.; De Angelis, F. Hyperbolic Meta-Antennas Enable Full Control of Scattering and Absorption of Light. Nano Lett. 2019, 19, $1851-1859$.

(20) Papadakis, G. T.; Fleischman, D.; Davoyan, A.; Yeh, P.; Atwater, H. A. Optical magnetism in planar metamaterial heterostructures. Nat. Commun. 2018, 9, 296.

(21) Kuttruff, J.; Garoli, D.; Allerbeck, J.; Krahne, R.; De Luca, A.; Brida, D.; Caligiuri, V.; Maccaferri, N. Ultrafast all-optical switching enabled by epsilon-near-zero modes in metal-insulator nanocavities. Commun. Phys. 2020, 3, 114.

(22) Caligiuri, V.; Pianelli, A.; Miscuglio, M.; Patra, A.; Maccaferri, N.; Caputo, R.; De Luca, A. Near- and Mid-Infrared Graphene-Based Photonic Architectures for Ultrafast and Low-Power Electro-Optical Switching and Ultra-High Resolution Imaging. ACS Applied Nano Materials 2020, 3, 12218-12230.

(23) Rashed, A. R.; Yildiz, B. C.; Ayyagari, S. R.; Caglayan, H. Hot electron dynamics in ultrafast multilayer epsilon-near-zero metamaterials. Phys. Rev. B: Condens. Matter Mater. Phys. 2020, 101, 165301.

(24) Sreekanth, K. V.; Alapan, Y.; ElKabbash, M.; Ilker, E.; Hinczewski, M.; Gurkan, U. A.; De Luca, A.; Strangi, G. Extreme sensitivity biosensing platform based on hyperbolic metamaterials. Nat. Mater. 2016, 15, 621-627.

(25) Carrara, A.; Maccaferri, N.; Cerea, A.; Bozzola, A.; De Angelis, F.; Proietti Zaccaria, R.; Toma, A. Plasmon Hybridization in Compressible Metal-Insulator-Metal Nanocavities: An Optical Approach for Sensing Deep Sub-Wavelength Deformation. Adv. Opt. Mater. 2020, 8, 2000609.

(26) Duncan, C.; Perret, L.; Palomba, S.; Lapine, M.; Kuhlmey, B. T.; de Sterke, C. M. New avenues for phase matching in nonlinear hyperbolic metamaterials. Sci. Rep. 2015, 5, 8983.
(27) Sun, Y.; Zheng, Z.; Cheng, J.; Sun, G.; Qiao, G. Highly efficient second harmonic generation in hyperbolic metamaterial slot waveguides with large phase matching tolerance. Opt. Express 2015, 23, 6370-6378.

(28) Vincenti, M. A.; Kamandi, M.; de Ceglia, D.; Guclu, C.; Scalora, M.; Capolino, F. Second-harmonic generation in longitudinal epsilonnear-zero materials. Phys. Rev. B: Condens. Matter Mater. Phys. 2017, 96, 045438.

(29) Wu, W.; Fan, L.; Zang, W.; Yang, X.; Zhan, P.; Chen, Z.; Wang, $Z$. Second harmonic generation enhancement from a nonlinear nanocrystal integrated hyperbolic metamaterial cavity. Opt. Express 2017, 25, 21342-21348.

(30) Vincenti, M. A.; de Ceglia, D.; Scalora, M. ENZ materials and anisotropy: enhancing nonlinear optical interactions at the nanoscale. Opt. Express 2020, 28, 31180-31196.

(31) Suresh, S.; Reshef, O.; Alam, M. Z.; Upham, J.; Karimi, M.; Boyd, R. W. Enhanced Nonlinear Optical Responses of Layered Epsilon-near-Zero Metamaterials at Visible Frequencies. ACS Photonics 2020, xx DOI: 10.1021/acsphotonics.0c01178.

(32) Shen, Q.; Jin, W.; Yang, G.; Rodriguez, A. W.; Mikkelsen, M. H. Active Control of Multiple, Simultaneous Nonlinear Optical Processes in Plasmonic Nanogap Cavities. ACS Photonics 2020, 7 (4), 901-907.

(33) Rogov, A.; et al. Simultaneous Multiharmonic Imaging of Nanoparticles in Tissues for Increased Selectivity. ACS Photonics 2015, 2, 1416-1422.

(34) Smith, A.; Mancini, M.; Nie, S. Second window for in vivo imaging. Nat. Nanotechnol. 2009, 4, 710-711.

(35) Welsher, K.; Liu, Z.; Sherlock, S. P.; Robinson, J. T.; Chen, Z.; Daranciang, D.; Dai, H. A route to brightly fluorescent carbon nanotubes for near-infrared imaging in mice. Nat. Nanotechnol. 2009, 4, 773-780.

(36) Malitson, H. Interspecimen Comparison of the Refractive Index of Fused Silica. J. Opt. Soc. Am. 1965, 55, 1205-1209.

(37) Tan, C. Z. Determination of refractive index of silica glass for infrared wavelengths by IR spectroscopy. J. Non-Cryst. Solids 1998, 223, 158-163.

(38) Chen, B.; Bruck, R.; Traviss, D.; Khokhar, A. Z.; Reynolds, S.; Thomson, D. J.; Mashanovich, G. Z.; Reed, G. T.; Muskens, O. L. Hybrid Photon-Plasmon Coupling and Ultrafast Control of Nanoantennas on a Silicon Photonic Chip. Nano Lett. 2018, 18, 610-617.

(39) Hache, F.; Ricard, D.; Flytzanis, C.; Kreibig, U. The Optical Kerr Effect in Small Metal Particles and Metal Colloids: The Case of Gold. Appl. Phys. A: Solids Surf. 1988, 47, 347-357.

(40) Conforti, M.; Della Valle, G. Derivation of third-order nonlinear susceptibility of thin metal films as a delayed optical response. Phys. Rev. B: Condens. Matter Mater. Phys. 2012, 85, 245423.

(41) Lysenko, O.; Bache, M.; Lavrinenko, A. Third-order susceptibility of gold for ultrathin layers. Opt. Lett. 2016, 41, 317320

(42) Boyd, R. W.; Shi, Z.; De Leon, I. The third-order nonlinear optical susceptibility of gold. Opt. Commun. 2014, 326, 74-79.

(43) Nesterov, M.; Schäferling, M.; Weber, K.; Neubrech, F.; Giessen, H.; Weiss, T. Line-current model for deriving the wavelength scaling of linear and nonlinear optical properties of thin elongated metallic rod antennas. J. Opt. Soc. Am. B 2018, 35, 1482-1489.

(44) Celebrano, M.; Locatelli, A.; Ghirardini, L.; Pellegrini, G.; Biagioni, P.; Zilli, A.; Wu, X.; Grossmann, S.; Carletti, L.; De Angelis, C.; Duò, L.; Hecht, B.; Finazzi, M. Evidence of Cascaded ThirdHarmonic Generation in Noncentrosymmetric Gold Nanoantennas. Nano Lett. 2019, 19, 7013-7020.

(45) Thyagarajan, K.; Rivier, S.; Lovera, A.; Martin, O. J. F. Enhanced second-harmonic generation from double resonant plasmonic antennae. Opt. Express 2012, 20, 12860-12865.

(46) Canfield, B. K.; Husu, H.; Laukkanen, J.; Bai, B.; Kuittinen, M.; Turunen, J.; Kauranen, M. Local Field Asymmetry Drives SecondHarmonic Generation in Noncentrosymmetric Nanodimers. Nano Lett. 2007, 7, 1251-1255. 
(47) Celebrano, M.; et al. Mode matching in multiresonant plasmonic nanoantennas for enhanced second harmonic generation. Nat. Nanotechnol. 2015, 10, 412-417.

(48) Gennaro, S. D.; Li, Y.; Maier, S. A.; Oulton, R. F. Double Blind Ultrafast Pulse Characterization by Mixed Frequency Generation in a Gold Antenna. ACS Photonics 2018, 5, 3166-3171.

(49) Sartorello, G.; Olivier, N.; Zhang, J.; Yue, W.; Gosztola, D. J.; Wiederrecht, G. P.; Wurtz, G.; Zayats, A. V. Ultrafast Optical Modulation of Second- and Third-Harmonic Generation from CutDisc-Based Metasurfaces. ACS Photonics 2016, 3, 1517-1522.

(50) Grzelczak, M.; Pérez-Juste, J.; Mulvaney, P.; Liz-Marzán, L. M. Shape control in gold nanoparticle synthesis. Chem. Soc. Rev. 2008, 37, 1783-1791.

(51) Liz-Marzán, L. M. Tailoring Surface Plasmons through the Morphology and Assembly of Metal Nanoparticles. Langmuir 2006, 22, 32-41.

(52) Grzelczak, M.; Vermant, J.; Furst, E. M.; Liz-Marzán, L. M. Directed Self-Assembly of Nanoparticles. ACS Nano 2010, 4, 35913605.

(53) Verre, R.; Maccaferri, N.; Fleischer, K.; Svedendahl, M.; Odebo Länk, N.; Dmitriev, A.; Vavassori, P.; Shvets, I. V.; Käll, M. Polarization conversion-based molecular sensing using anisotropic plasmonic metasurfaces. Nanoscale 2016, 8, 10576-10581.

(54) Zubritskaya, I.; Maccaferri, N.; Inchausti Ezeiza, X.; Vavassori, P.; Dmitriev, A. Magnetic Control of the Chiroptical Plasmonic Surfaces. Nano Lett. 2018, 18, 302-307.

(55) Fredriksson, H.; Alaverdyan, Y.; Dmitriev, A.; Langhammer, C.; Sutherland, D. S.; Zäch, M.; Kasemo, B. Hole-Mask Colloidal Lithography. Adv. Mater. 2007, 19, 4297-4302.

(56) Biagioni, P.; Brida, D.; Huang, J.-S.; Kern, J.; Duò, L.; Hecht, B.; Finazzi, M.; Cerullo, G. Dynamics of Four-Photon Photoluminescence in Gold Nanoantennas. Nano Lett. 2012, 12, 29412947.

(57) Bouhelier, A.; Bachelot, R.; Lerondel, G.; Kostcheev, S.; Royer, P.; Wiederrecht, G. P. Surface Plasmon Characteristics of Tunable Photoluminescence in Single Gold Nanorods. Phys. Rev. Lett. 2005, 95, 267405.

(58) Bouhelier, A.; Beversluis, M. R.; Novotny, L. Characterization of nanoplasmonic structures by locally excited photoluminescence. Appl. Phys. Lett. 2003, 83, 5041.

(59) Knittel, V.; Fischer, M. P.; de Roo, T.; Mecking, S.; Leitenstorfer, A.; Brida, D. Nonlinear Photoluminescence Spectrum of Single Gold Nanostructures. ACS Nano 2015, 9, 894-900.

(60) Dmitriev, V. G.; Gurzadyan, G. G.; Nikogosyan, D. N. Handbook of Nonlinear Optical Crystals, 3rd ed.; Springer, 1999.

(61) Boyd, R. Nonlinear Optics, 4th ed., 2020, Elsevier.

(62) Garrett, C.; Robinson, F. Miller's phenomenological rule for computing nonlinear susceptibilities. IEEE J. Quantum Electron. 1966, 2, 328-329 (1966).

(63) Miller, R. C. Optical second harmonic generation in piezoelectric crystals. Appl. Phys. Lett. 1964, 5, 17-19.

(64) Finazzi, M.; Biagioni, P.; Celebrano, M.; Duò, L. Selection rules for second-harmonic generation in nanoparticles. Phys. Rev. B: Condens. Matter Mater. Phys. 2007, 76, 125414.

(65) Lodewijks, K.; Miljkovic, V.; Massiot, I.; Mekonnen, A.; Verre, R.; Olsson, E.; Dmitriev, A. Multiscale conformal pattern transfer. Sci. Rep. 2016, 6, 28490.

(66) Staedler, D.; et al. Harmonic Nanocrystals for Biolabeling: A Survey of Optical Properties and Biocompatibility. ACS Nano 2012, 6, $2542-2549$.

(67) Grange, R.; Lanvin, T.; Hsieh, C.-L.; Pu, Y.; Psaltis, D. Imaging with second-harmonic radiation probes in living tissue. Biomed. Opt. Express 2011, 2, 2532-2539.

(68) Dreaden, E. C.; Alkilany, A. M.; Huang, X.; Murphy, C. J.; ElSayed, M. A. The golden age: Gold nanoparticles for biomedicine. Chem. Soc. Rev. 2012, 41, 2740-2779.

(69) Lagerwall, J. P. F.; Schütz, C.; Salajkova, M.; Noh, J.; Park, J. H.; Scalia, G.; Bergström, L. Cellulose nanocrystal-based materials: from liquid crystal self-assembly and glass formation to multifunctional thin films. NPG Asia Mater. 2014, 6, No. e80.

(70) Autere, A.; Jussila, H.; Dai, Y.; Wang, Y.; Lipsanen, H.; Sun, Z. Nonlinear Optics with 2D Layered Materials. Adv. Mater. 2018, 30, 1705963.

(71) Indukuri, S. R. K. C.; Frydendahl, C.; Bar-David, J.; Mazurski, N.; Levy, U. WS 2 Monolayers Coupled to Hyperbolic Metamaterial Nanoantennas: Broad Implications for Light-Matter-Interaction Applications. ACS Appl. Nano Mater. 2020, 3, 10226-10233.

(72) Rakić, A. D.; Djurisic, A. B.; Elazar, J. M.; Majewski, M. L. Optical properties of metallic films for vertical-cavity optoelectronic devices. Appl. Opt. 1998, 37, 5271-5283.

(73) https://www.comsol.it/model/scatterer-on-substrate-14699. 